Alkalen reflü gastrit tanısı ile ursodeoksikollk asit tedavisi almış hastaların tedavi öncesi ve sonrası klinik, endoskopik ve histolojik bulgularının değerlendirilmesi

Comparison of clinical, endoscopic, and histological features of patients diagnosed with alkaline reflux gastritis before and after ursodeoxycholic acid treatment

\title{
Halil RAKICI
}

Recep Tayyip Erdoğan Üniversitesi Tip Fakültesi, Gastroenteroloji Bilim Dalı, Rize

Giriş ve Amaç: Alkalen reflü gastrit asidik olmayan safranın mide içine reflüsüne verilen isimdir. Opere midede sık görülmekle birlikte cerrahi girişim geçirmeyen hastalarda da duodenogastrik antifeflü mekanizmalardaki bozukluk sonucunda ortaya çıkmaktadır. Medikal tedavisinde çeşitli ilaçlar kullanılmaktadır. Biz bu çalışmada cerrahi girişim geçirmemiş hastalarda ursodeoksikolik asitin klinik, endoskopik ve histolojik etkilerini incelemeyi amaçladık. Gereç ve Yöntem: Endoskopik inceleme sonucu alkalen reflü gastrit saptanan 72 hastaya ursodeoksikolik asit tedavisi verildi. Iki aylik tedavi sonunda klinik, endoskopik ve histolojik bulgular değerlendirildi. Klinik değerlendirme 72 hastada, endoskopik ve histolojik değerlendirme ise kontrol endoskopi yaptıran 61 hasta üzerinden yapıldı. Bulgular: Hastaların 38'i (\%52.7) kadın, 34'ü (\%47.3) erkekti. En küçük yaș 19, en büyük yaş 81 olup, ortalama yaş 50 idi. Tedavi sonrası 72 hastanın 54'ü (\%75) tedaviden fayda gördügünü bildirdi. Kontrol endoskopi yapılan 61 hastanın 45'inde (\%73.7) endoskopik tanı alkalen reflü gastrit olarak bildirilmedi. Kontrol histolojik inceleme yapılan 61 hastanın 6'sında (\%9.9) kronik gastrit ve inflamasyon yerine normal mukoza saptandı. Sonuç: Ursodeoksikolik asit tedavisi verilen hastaların \% 75'inin klinik olarak tedaviden fayda gördügü saptandı. Endoskopik olarak tedavi sonrası hastaların \%73.7'sinde alkalen reflu gastrit saptanmadi. Histolojik olarak hastaların ancak \%9.9'unda histolojik bulguların düzeldiği görüldü. Bu bulgularla alkali reflü gastrit tedaviside ursodeoksikolik asitin klinik ve endoskopik bulguların düzelmesinde etkili, histolojik bulguları düzeltmede ise etkisiz olduğunu söylemek mümkündür.

Anahtar kelimeler: Alkalen reflü, gastrit, ursodeoksikolik asit
Backgrounds and Aims: Alkaline reflux gastritis is characterized by non-acidic flow from the duodenum to the stomach. It is seen very commonly among operated patients. It can also be seen among non-operated patients, due to a defect in duodenogastric antireflux mechanisms. Various medications are used for its treatment. We aimed to analyze the clinical, endoscopic, and histological effects of ursodeoxycholic acid in non-operated patients in this study. Materials and Methods: A total of 72 patients who were diagnosed with alkaline reflux gastritis as a result of endoscopic imagining were treated with ursodeoxycholic acid. Clinical, endoscopic, and histological features were analyzed after 2 months of treatment. A total of 72 patients were analyzed clinically, and 61 patients who had control endoscopy were analyzed endoscopically and histologically. Results: Of the total, 38 patients were female and 34 were male. The youngest and oldest patients were 19 and 81 years of age, respectively. The average age was 50 years. After treatment, 54 patients out of 72 reported benefits. Sixteen patients out of 61 who had control endoscopy were diagnosed endoscopically with alkaline reflux gastritis. Normal mucosa was observed instead of chronic gastritis and inflammation in 6 out of 61 patients who had control histological examination. Conclusion: In all, 75\% of the patients who were treated with ursodeoxycholic acid recovered clinically. Altogether, $73.7 \%$ of patients recovered endoscopically. Only $9.9 \%$ of patients recovered histologically. In conclusion, ursodeoxycholic acid treatment is effective clinically and endoscopically but is ineffective histologically.

Key words: Alkaline reflux, gastritis, ursodeoxycholic acid

mekanizmaların yetersizliği sonucu olduğu düşünülmektedir. Safra ve duodenal içeriğin pH'sı mide sıvısına göre daha yüksek (asidite daha düşük) olduğu için alkalen reflü gastrit olarak da adlandırılmaktadır. Alkalen reflü gastrit tanısı koymak bazen midesinde safra saptanan hastalarm herhangi bir semptomu olmaması yüzünden sorunlu olabilmektedir. Midede safra varlığı direkt endoskopik olarak, Bilitec yöntemi veya sintigrafik olarak saptanabilmektedir. Ancak bu yöntemlerle gastropatinin varllğı gösterilemez. Tanı için histopatolojik inceleme gereklidir (5-12). Tedavide proton pompa inhibitörleri (PPI), antasitler ve sukralfatın faydalı olduğunu bildiren yayınlar vardır (13-18). Ursodeoksikolik asitin ve kolestraminin etkisi bazı soru işaretleri taşımaktadır $(15,18)$. Medikal tedavi ilk tercih edilecek tedavidir. Cerrahi tedavi 
zorunlu hallerde yapılmalıdır (14). Seçilen yöntem genellikle Roux en Y diversiyon operasyonudur. Tedavide PPİ ve antasitler kullanılmış hem semptomatik ve hem de histopatolojik anomalilerin düzeldiği görülmüştür. Ayrıca endoskopik olarak safra reflüsünün azaldığı saptanmıștır. Sukralfat da bazı hastalarda başarı ile kullanılmıştır. Ursodeoksikolik asit ve kolestramin de tedavide kullanılmakta birlikte etkileri net olarak belirlenmemiştir. Daha önceki yayınlarda klinik ve endoskopik düzelme bildirilirken histolojik bulgularda değişiklik olmadığı bildirilmektedir. Tedaviye dirençli vakalarda cerrahi diversiyon operasyonu yapılmaktadır. Biz bu çalışmada opere olmamıs primer safra reflüsü olan hastalarda ursodeoksikolik asit tedavisinin klinik, endoskopik ve histolojik etkilerini değerlendirmek istedik.

\section{GEREC ve YÖNTEM}

Endoskopik olarak alkalen reflü gastrit tanısı olan ve ursodeoksikolik asit tedavisi verilen 72 hasta değerlendirildi. Hastalar 2015-2016 yılları arası Recep Tayyip Erdoğan Üniversitesi Gastroenteroloji Endoskopi Ünitemize bașvuranlar arasından seçildi. Bunlardan 61 tanesine 4 haftalık ursodeoksikolik asit tedavi sonrası kontrol endoskopi yapıldı. Onbir hasta kontrol endoskopi yaptırmadı. Daha önce PPİ alanlara tedaviye devam etmesi istendi. PPI almayanlara ise ilave edilmedi. Helicobacter pylori varlığı dikkate alınmadı. Tedavi süresince eradikasyon tedavisi verilmedi. Histolojik incelemeler rutin işleyiş içinde değişik patologlar tarafından daha öncesi bilinmeden (kör olarak) değerlendirildi. Recep Tayyip Erdoğan Üniversitesi Etik Kurul onayı alındı. Istatistiki analiz için SPSS programı kullanıldı. Tanımlayıcı istatistikler uygulandı.

\section{BULGULAR}

Çalışmaya alınan hastaların 38'i (\%52.7) kadın, 34’ü (\%47.3) erkekti. En küçük yaş 19, en büyük yaş 81 olup ortalama yaş 50 idi. Tedavi sonrası 72 hastanın 54'ü (\%75) tedaviden fayda gördüğünü bildirdi. Hastalardan kontrol endoskopi yapılan 61 hastanın 16'sında (\%26.3) endoskopik tanı yine alkalen reflü gastrit olarak geldi. Kontrol histolojik inceleme yapilan 61 hastanın 6'sında (\%9.9) kronik gastrit ve inflamasyon yerine normal mukoza saptandı. Diğer 55 hastada histolojik bulgularda değişiklik olmadı. Ilk histolojik incelemede 52 hastada kronik gastrit ve inflamasyon bulguları, 9 hastada intestinal metaplazi, 6 hastada foveolar hiperplazi, 5'inde lenfoid folikül saptandı. Bulgularımız (Tablo 1,2,3) ve (Resim $1,2,3)$ 'te gösterilmiştir.

Tablo 1. Temel özellikler

$\begin{array}{ll}\text { Yaş } & 19-81 \text { (Ortalama 50) } \\ \text { Cinsiyet } & 38 \text { kadın }(\% 52,7), 34 \text { erkek (\%47.3) }\end{array}$

$\begin{array}{lcc}\text { Tablo 2. Temel bulgular } & \\ & \begin{array}{c}\text { Tedaviye } \\ \text { Cevap Veren }\end{array} & \begin{array}{c}\text { Tedaviye } \\ \text { Cevap Vermeyen }\end{array} \\ \text { Klinik bulgular } & 54(\% 75) & 18(\% 25) \\ \text { Endoskopik bulgular } & 45(\% 73.7) & 16(\% 26.3) \\ \text { Histolojik bulgular } & 6(\% 9.9) & 55(\% 90.1)\end{array}$

Tablo 3. Histolojik bulgular

\section{Başlangıç Bulguları Kontrol Bulguları}

Foveolar hiperplazi $\quad 72 / 6(\% 8.3) \quad 61 / 6(\% 9.8)$

Kronik gastrit, $\quad 72 / 52(\% 72.2) \quad 61 / 46(\% 75.4)$ Inflamasyon

Lenfoid follikül

$72 / 5(\% 6.9)$

$61 / 5(\% 8.1)$

Intestinal metaplazi

$72 / 9$ (\% 12.5)

$61 / 9(\% 14.7)$

Normal mukoza

$72 / 0(\% 0)$

$61 / 6(\% 9.8)$

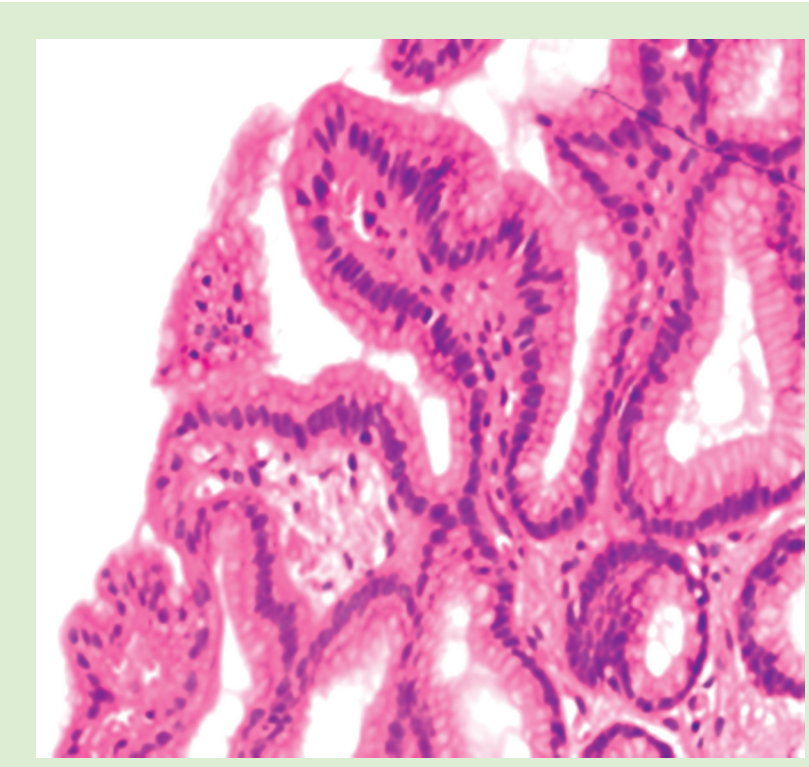

Resim 1. Foveolar hiperplazi (Hematoksilen\&Eozin).

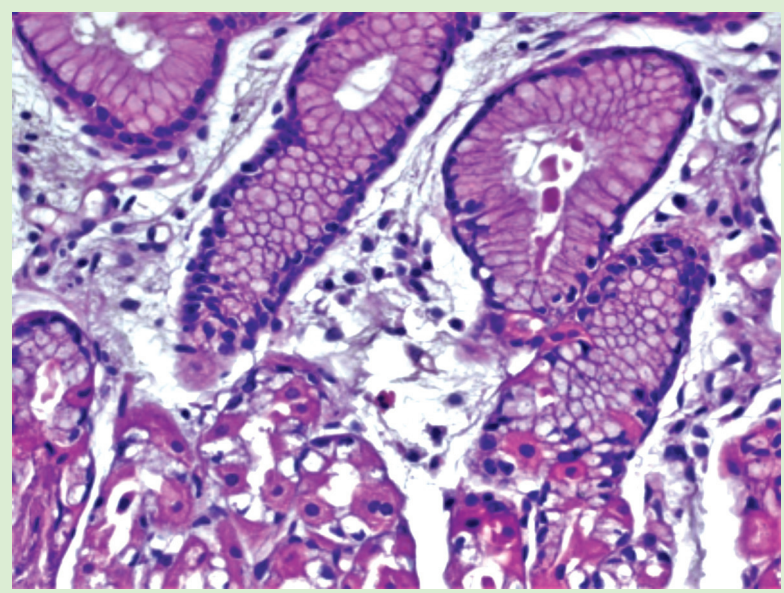

Resim 2. Kronik inflamasyon (Hematoksilen\&Eozin) 


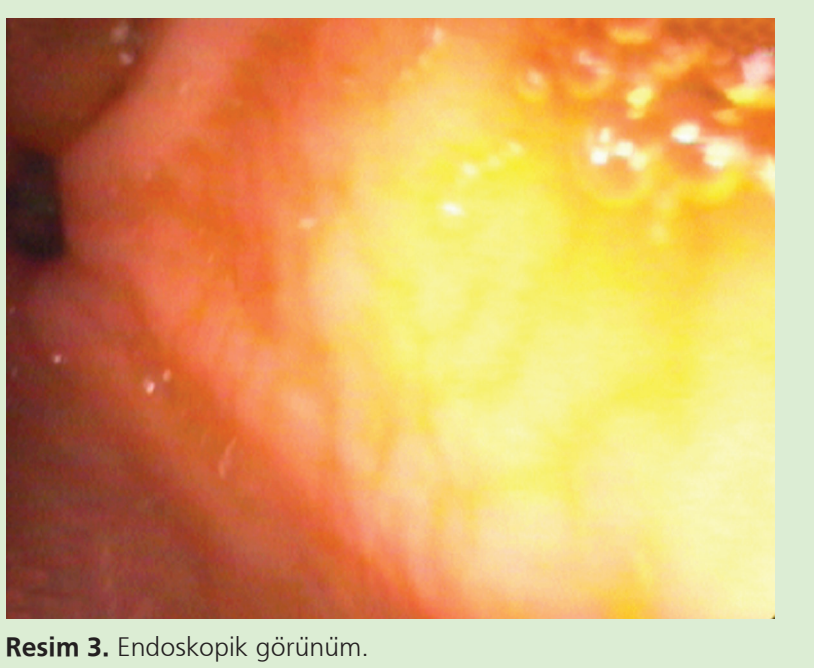

\section{TARTIŞMA}

Cerrahi girişim olmadan ortaya çıkan primer safra reflüsünün sebebi net olarak bilinmemekle beraber duodenogastrik reflüyü önleyici mekanizmalardaki yetersizlik sorumlu tutulmaktadır. Safra içeriği mide mukozası için agressif bir faktör olarak davranmakta ve kronik inflamasyona yol açmaktadır. Tipik histolojik bulgular foveolar hiperplazi, kronik inflamasyon ve intestinal metaplazi şeklinde görülebilmektedir. Safra reflüsü veya diğer bir tanımlamayla alkalen reflü gastritte çeşitli tedavi seçenekleri denenmiş olup PPİ, antasit ve sukralfatın etkili olduğunu bildiren yayınlar mevcuttur. Usodeoksikolik asitin tedavide kullanılmasına ilişkin bilgilerimiz 1986 yılına ait bir çalışmaya dayanmaktadır. O tarihten bu yana bu konudaki yayınlarda bir duraklama göze çarpmaktadır. $\mathrm{Bu}$ nedenle bu konudaki bilgilerimizin güncellenmesine ihtiyaç olduğunu düşünmekteyiz. Ülkemizde Sağlık Uygulama Talimatı (SUT) ile alkalen reflü tanısıyla ursodeoksikolik asit geri ödeme kapsamındadır. Çalışmamızda hastaların büyük çoğunluğunda semptomlarda tedavi ile anlamlı iyileşme görüldüğü saptanmıştır. Aynı şekilde endoskopik olarak tedavi sonrası yapılan kontrol endoskopisinde de safra reflüsünde anlamlı azalma gözlenmiştir. Safra reflüsündeki azalmanın nedeni net olarak bilinmese de safra içeriğinin ursodeoksikolik asit tedavisi ile değişmesine bağlanmaktadır. Midede safra varlığı endoskopik olarak, Bilitec yöntemi ve sintigrafik olarak gösterilebilir. Bu yöntemlerin birbirlerine benzer etkinlikte olduğu bildirilmektedir (1-3,10-12). Histolojik bulgular değerlendirildiğinde iki aylık tedavi sonrası 61 hastanın kronik inflamasyon saptanan altısında kontrol incelemede normal mukoza saptanması anlamlı değildir (\%9.9). Bu anlamda bugün güncellenen bilgilerimiz otuz yıl öncesinden farklı değildir. Çalışmamızda histolojik olarak inflamasyona yol açan veya tedaviye katkıda bulunan diğer faktörler (Helicobacter pylori varlığı ve PPİ kullanımı) sabit tutularak değerlendirilmeye katılmadı. Bu gruptaki hastaların ursodeoksikolik asit tedavisi bitene kadar mevcut durumları korundu. Diğer bir deyişle PPI kullanan hastalar kullanmaya devam etti ve Helicobacter pylori pozitif ise tedavi sonuna kadar eradikasyon tedavisi verilmedi. Bu sayede oluşabilecek histolojik değişikliklerin ursodeoksikolik asit tedavisinin sonucunda ortaya çıkması sağlanmaya çalışıldı. Histolojik preparatlar daha önceki histolojik bulgulardan haberdar olmayan patologlarca değerlendirildi. Hastalarda anlamlı bulunan klinik ve endoskopik iyileşmenin hekimin subjektif gözlemine bağlı olması çalışmamızın zayıf yönünü oluşturduğu söylenebilir. Histolojik bulguların rapor edilmesi patologlarca kronik gastrit, kronik aktif gastrit, inaktif gastrit, hafif gastrit ve kronik inflamasyon gibi farklı tanımlamalar şeklinde olmuştur. Hastaların endoskopi raporları farklı endoskopistler tarafından verilmiştir. Histolojik bulgu olarak foveolar hiperplazi literatürde başlıca bulgu olarak belirtilmesine karşın bu çalışmamızda patologlar tarafından en sık rapor edilen bulgu olmamıştır. Tedavide PPI'lar, antiasitler, sukralfalt, ursodeoksikolik asit ve kolestramin kullanılmaktadır. Ancak bu konuda yapılmıs geniş çaplı randomize yayınlar yeterli değildir. Prostaglandin analogları etkisiz bulunmuştur. Literatürde ursodeoksikolik asit tedavisi ile semptomlarda ve endoskopik bulgularda iyileşme gözlendiği halde histolojik iyileşme gözlenmemektedir. Tersine sukralfatla histolojik düzelme olmasına rağmen semptomlarda veya endoskopik bulgularda düzelme olmamaktadır. PPI ve antiasit kombinasyonu hem endoskopik hem de histolojik bulgularda düzelme sağlamaktadır (13-18). Bizim bulgularımız da literatüre paralel bulunmuştur. Histolojik bulgularda düzelme olmaması, ursodeoksikolik asit tedavisinin ancak ilacın alındığı sürede etkili olduğu, kalıcı bir düzelme sağlamadığı sonucuna varmamıza yol açabilecek bir bulgudur.

Sonuç olarak bu bulgularla alkalen reflü gastrit tedavisinde ursodeoksikolik asitin klinik ve endoskopik bulguları düzeltmede etkili, histolojik bulguları düzeltmede ise etkisiz oldugunu söyleyebiliriz.

\section{ACIKLAMA}

Yazar bu yazının yayınlanmasında herhangi bir çıkar çatışması veya finansal destek olmadığını beyan eder.

\section{TEȘEKKÜR}

Yazının patolojik bulguların değerlendirilmesinde katkısı bulunan Sayın Doç. Dr. Recep Bedir'e ve istatistiki bulgulara katkısı bulunan Sayın Dr. Başar Erdivanlı'ya teşekkürü bir borç bilirim. 


\section{KAYNAKLAR}

1. Feldman M, Edward LL, "Gastritis" in Sleisenger and Fordtran's Gastrointestinal and Liver Disease. Pathophysiology/Diagnosis/Management, M. Feldman, S. F. Lawrence, andJ. B. Lawrence, Eds., pp.880-881, Saunders, Philadelphia, Pa,USA, 10th edition, 2015.

2. Pamela J Jensen, Mark Feldman. Acute hemorrhagic erosive gastropathy and chronic chemical gastropathy.Literature review current through: Apr 2017. I This topic last updated: Dec 17, 2015. UpToDate.

3. Niemelä S. Duodenogastric reflux in patients with upper abdominal complaints or gastric ulcer with particular reference to reflux-associated gastritis. Scand J Gastroenterol Suppl 1985;115:1-56.

4. Dixon MF, O'Connor HJ, Axon AT, et al. Reflux gastritis: distinct histopathological entity? J Clin Pathol 1986;39:524-30.

5. Weinstein WM, Buch KL, Elashoff J, et al. The histology of the stomach in symptomatic patients after gastric surgery: a model to assess selective patterns of gastric mucosal injury. Scand J Gastroenterol Suppl 1985;109:77-89.

6. Nakamura M, Haruma K, Kamada T, et al. Duodenogastric reflux is associated with antral metaplastic gastritis. Gastrointest Endosc 2001;53:53-9.

7. Orchard R, Reynolds K, Fox B, et al. Effect of lysolecithin on gastric mucosal structure and potential difference. Gut 1977;18:457-61.

8. Eastwood GL. Effect of pH on bile salt injury to mouse gastric mucosa. A light- and electron-microscopic study. Gastroenterology 1975;68:1456-65.

9. Karttunen T, Niemelä S. Campylobacter pylori and duodenogastric reflux in peptic ulcer disease and gastritis. Lancet 1988;1:118
10. Bondurant FJ, Maull KI, Nelson HS Jr, et al. Bile reflux gastritis. South Med J 1987;80:161-5.

11. Stein HJ, Smyrk TC, DeMeester TR, et al. Clinical value of endoscopy and histology in the diagnosis of duodenogastric reflux disease. Surgery 1992;112:796-803.

12. Niemelä S, Karttunen T, Heikkilä J, Lehtola J. Characteristics of reflux gastritis. Scand J Gastroenterol 1987;22:349-54.

13. Chen H, Li X, Ge Z, et al. Rabeprazole combined with hydrotalcite is effective for patients with bile reflux gastritis after cholecystectomy. Can J Gastroenterol 2010;24:197-201.

14. Davidson ED, Hersh T. The surgical treatment of bile reflux gastritis: a study of 59 patients. Ann Surg 1980;192:175-8.

15. Stefaniwsky AB, Tint GS, Speck J, et al. Ursodeoxycholic acid treatment of bile reflux gastritis. Gastroenterology 1985;89:1000-4.

16. Buch KL, Weinstein WM, Hill TA, et al. Sucralfate therapy in patients with symptoms of alkaline reflux gastritis. A randomized, double-blind study. Am J Med 1985;79:49-54.

17. Nicolai JJ, van de Stadt J, Tytgat GN. Double-blind crossover trial of prostaglandin E2 in postgastrectomy reflux gastritis. Dig Dis Sci 1986;31:1281-6.

18. Nicolai JJ, Speelman P, Tytgat GN, et al. Comparison of the combination of cholestyramine/alginates with placebo in the treatment of postgastrectomy biliary reflux gastritis. Eur J Clin Pharmacol 1981;21:189-94. 